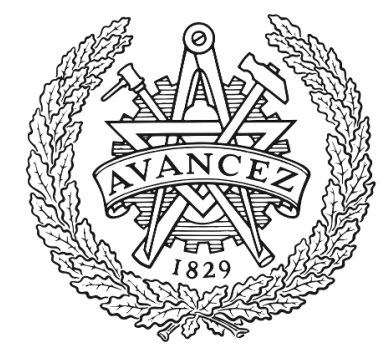

\title{
CHALMERS
}

UNIVERSITY OF TECHNOLOGY

\section{Measurement Technique to Emulate Signal Coupling Between Power Amplifiers}

Downloaded from: https://research.chalmers.se, 2023-04-26 13:06 UTC

Citation for the original published paper (version of record):

Nopchinda, D., Buisman, K. (2018). Measurement Technique to Emulate Signal Coupling Between Power Amplifiers. IEEE Transactions on Microwave Theory and Techniques, 66(4): 2034-2046. http://dx.doi.org/10.1109/TMTT.2017.2786274

N.B. When citing this work, cite the original published paper.

(O2018 IEEE. Personal use of this material is permitted.

However, permission to reprint/republish this material for advertising or promotional purposes 
This is the accepted version of a work that was accepted for publication in the following source: IEEE Transactions on Microwave Theory and Techniques

\section{Measurement Technique to Emulate Signal Coupling Between Power Amplifiers \\ By D. Nopchinda and K. Buisman}

This file was downloaded from: the Chalmers Research website https://research.chalmers.se

Copyright (C) 2018, IEEE

Notice: Changes introduced as a result of the publishing process, such as editing and formatting may not be reflected in this document. For the final version of this work, please refer to the published source:

https://doi.org/10.1109/TMTT.2017.2786274

N.B. When citing this work, cite the final version. 


\title{
Measurement Technique to Emulate Signal Coupling Between Power Amplifiers
}

\author{
Dhecha Nopchinda, Student Member, IEEE, and Koen Buisman, Member, IEEE
}

\begin{abstract}
A measurement technique to emulate coupling between power amplifiers (PA) such as that in an antenna array is presented. The case the technique aims to emulate is referred to as the target array. The technique provides emulation of the distorted output signal for each PA under the coupling effect without the requirement for constructing the target physical coupling network or antenna array. Furthermore, given that the target array contains identical elements or PAs, transmitting either identical or different signals, the technique merely requires one PA as device under test (DUT) to produce all output signals. The technique has direct connection to active load-pull, and aims to present the output of the DUT with corresponding timevarying impedances of each transmission path in the target array. The emulated output signals can then be analyzed, for example, in terms of adjacent channel power ratio, error vector magnitude, and normalized mean square error. Such measurement technique provides insight into the distortion and the impairment generated in the target array without the requirement to realize an actual array, and can be used, as an example, during the design stage of an array. The technique is theoretically motivated. The procedure is thoroughly described. The technique is experimentally demonstrated and verified under various usage cases and scenarios. Subsequent comparison to conventional active load-pull is provided.
\end{abstract}

Index Terms-Active load-pull, antenna array, coupling effect, distortion, emulation, measurement technique, power amplifier.

\section{INTRODUCTION}

A NTENNA ARRAYS are used in modern communication systems for multiple purposes, such as, beamforming, and addressing bandwidth limitations [1]. In future systems, e.g., Massive multiple-input multiple-output (MIMO), large antenna arrays with high number of antennas, power amplifiers (PA), and transmission paths are being used [2]. Furthermore, with higher operating radio frequency (RF) in the millimeter-wave (mmWave) bands, low-cost integrated designs are of particular interest [3]. However, such designs are susceptible to the so-called "antenna array coupling effects", where the interactions between elements in the array

The results in this paper are partly the result of the project MET5G Metrology for $5 \mathrm{G}$ communications. This project has received funding from the EMPIR programme co-financed by the Participating States and from the European Union's Horizon 2020 research and innovation programme.

This paper is an expanded version from the 2017 International Microwave Conference, Honolulu, HI, 4-9 June 2017.

D. Nopchinda, and K. Buisman are with the Microwave Electronics Laboratory, Department of Microtechnology and Nanoscience, Chalmers University of Technology, Gothenburg SE-412 96, Sweden (e-mail: dhecha@chalmers.se; buisman@chalmers.se). result in sub-optimal behavior and characteristics, e.g., reduced efficiency, and worsened level of distortion to the transmitted signal [4].

One main principle for antenna array coupling is the modulated signal at the output of one antenna is coupled into the output of the other antennas. i.e., RF outputs coupling. This results in variation of the output reflection coefficient of each power amplifier (PA), which in turn results in reduction of PA's efficiency and distortion. Typically, this effect is suppressed using isolators to eliminate such interaction. However due to its bulk and cost, in future communication system where the emphasis is in increasing integration, employing isolators may no longer be a viable or costeffective option.

Techniques have been developed to model antenna coupling in order to study its effect through estimation of parameters [5]. In this work, we instead propose a measurement setup paradigm along with experimental procedure in order to present the device under test (DUT) with time-varying reflection coefficient, determined by an S-parameter network which in turn represents RF outputs coupling in a given antenna array. The measurement technique provides this without the need for construction of an actual array. It also has minimal setup requirement of one DUT per identical group of PAs. Such technique is useful for studying the distortion generated in an array under the intended use-cases, for example, during the design stage.

This emulation technique has been proposed in [6] and [7]. However, work in [6] neglects proper calibration to the DUT's reference plane, rendering the technique unsuitable for complete S-parameter network representation. Furthermore, the PA's distortion under $50 \Omega$ loading condition was not taken into account; hence, the emulated results were not exclusive to the distortion from antenna array coupling. This effectively results in reduced dynamic range of the measurement. Work in [7] has addressed these problems, and in addition used realistic transmitted signals. However, it lacks the proper visualization and demonstration of the emulated reflection coefficient, the proper justification of its assumptions, and a comparison of the technique with traditional active load-pull. The work in this paper not only addresses these shortcomings, but also serves as a complete guide to the proposed measurement technique containing thorough analysis, motivation, and detailed explanation of the procedure. Furthermore, a new verification of the proposed technique is provided along with a section dedicated to discuss 
the pitfalls which could result in discrepancies users may wish to avoid, depending on the desired level of accuracy.

The organization of this paper is as follows. In Section II, generic interaction of PAs in an antenna array in terms of signal coupling is derived and discussed. Clear motivation and connection to the proposed technique is then given. In Section III, the experimental setup, the emulation method, and the experimental procedure are thoroughly described. In Section IV, verification cases under different operational scenarios of a two-element antenna array are motivated and described. The results and comparison between emulation and verification are subsequently analyzed in Section V. Comparisons with conventional active load-pull is discussed in Section VI. Section VII contains verification with a three-element antenna array under one usage scenario. The discussion on pitfalls of the technique supported by the marginal discrepancies between the emulation and the verification is provided in Section VIII. The paper is concluded and summarized in Section IX.

\section{THEORY}

This section analyzes an arbitrary antenna array using linear representation. Interactions at the RF outputs between transmission paths and their elements are considered in terms of reflection coefficients, S-parameter network, and incident and reflected waves.

\section{A. Active Load-pull Techniques}

Active load-pull techniques are often employed, since they circumvent the reduced reachable reflection coefficient associated with the losses in a passive load-pull system. Furthermore, active load-pull offers the possibility to use wideband modulated signals with fully controlled reflection coefficient as a function of frequency at a reference plane [8]. In Fig. 1(a), a conventional single tone load-pull system is shown. The phase and amplitude of the injected signal control the reflection coefficient. This can be extended to fully modulated signals as indicated in Fig. 1(c).

When extending to multi-port load-pull, as shown in Fig. 1(b), it becomes clear that coupling effects can be handled in the following manner. If the output $b_{2}$ waves are known from measurement, using knowledge of the coupling enables calculation of the to be injected $a_{2}$ waves needed to represent the correct coupling. Furthermore, note that, if the DUTs are identical, the same hardware is repeated, as indicated by the symmetry line. This implies that multiport load-pull can be done, using only one set of hardware, through splitting out in time domain. Both of these methods will be demonstrated in this paper.

\section{B. Generic Array with Signal Coupling}

In communication system, under operating condition, the transmitting modulated wide-band signal at the output of a given antenna is coupled onto the output of other antennas. These coupled signals then propagate into the corresponding transmission paths, which in turn vary the output reflection coefficients seen by respective PAs as a function of time. This

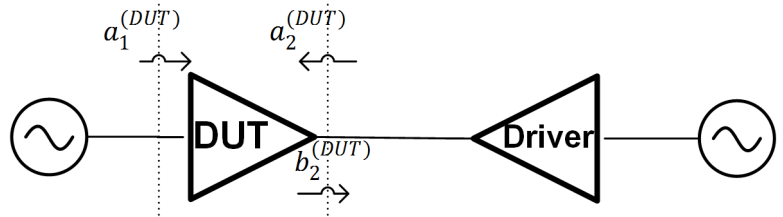

(a)
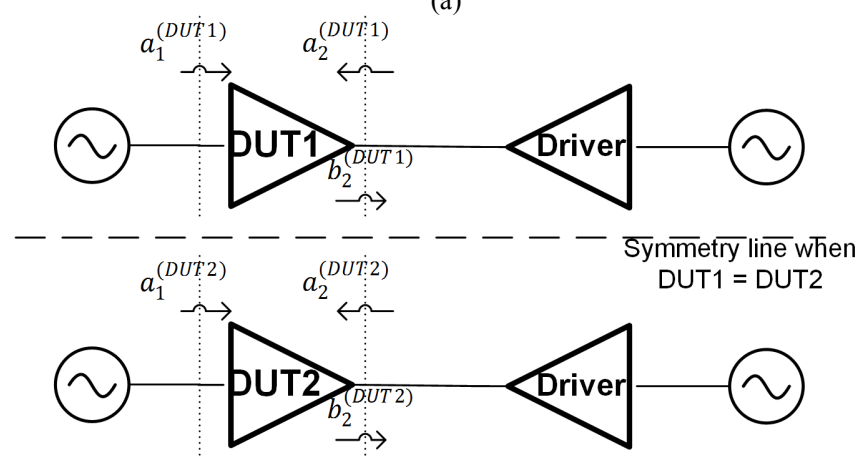

(b)

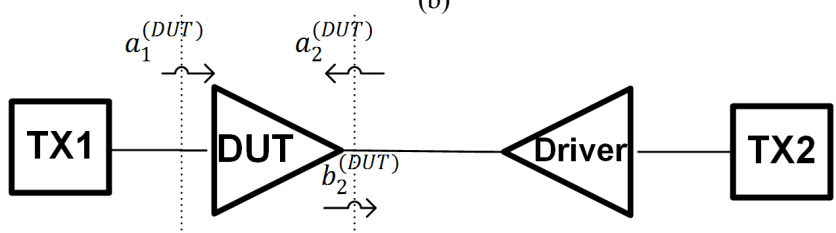

(c)

Fig. 1. Active load-pull techniques: (a) single tone load-pull, (b) multi-port load-pull, and (c) modulated signal load-pull.

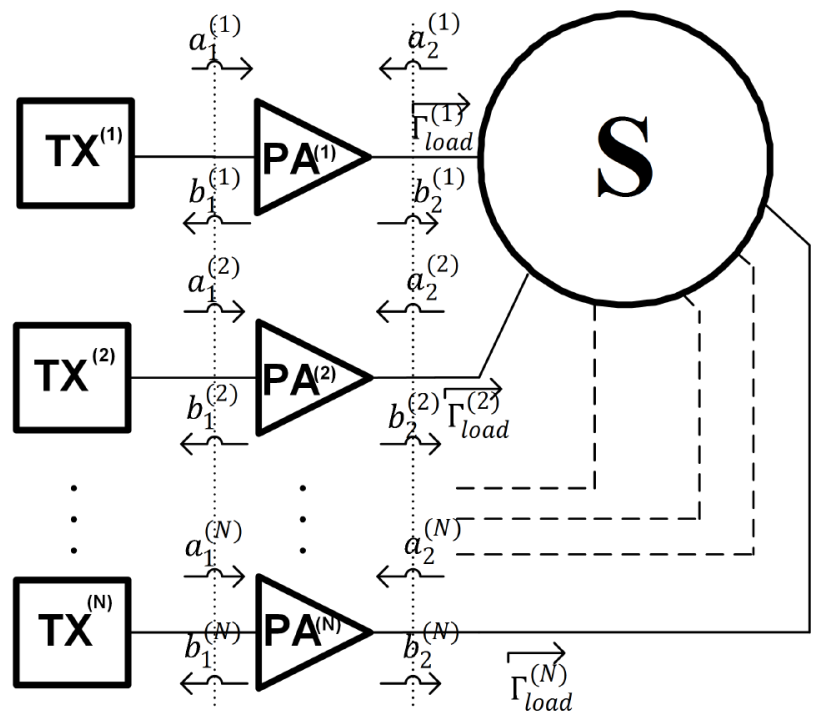

Fig. 2. Antenna array with $\mathrm{N}$ transmission paths whose coupling is defined by the S-parameter network, $\mathbf{S}$.

phenomenon happens simultaneously to all transmission paths, resulting in undesirable effects including distortion of the transmitted signal, reduction in power efficiency, and reduction in spectral efficiency [4].

An arbitrary antenna array including $\mathrm{N}$ antennas, N PAs, and $\mathrm{N}$ transmitters (TX) is shown in Fig. 2. RF output coupling between transmission paths is defined by a deterministic S-parameter network, denoted as $\mathbf{S}$, which can be represented as 


$$
\mathbf{S}=\left[S_{i j}\right]_{i, j \in 1,2, \ldots, N}=\left[\begin{array}{cccc}
S_{11} & S_{12} & \cdots & S_{1 N} \\
S_{21} & S_{22} & \cdots & S_{2 N} \\
\vdots & \vdots & \ddots & \vdots \\
S_{N 1} & S_{N 2} & \cdots & S_{N N}
\end{array}\right],
$$

where $S_{i j}, i \neq j$ represents the coupling factor, and $S_{i j}, i=j$ represents the reflection coefficient of a given transmission path. At the output of the $i^{\text {th }} \mathrm{PA}$, the reflection coefficient, $\Gamma_{\text {load }}^{(i)}$, is affected and destabilized due to these factors, and can be represented as

$$
\Gamma_{\text {load }}^{(i)}=\frac{a_{2}^{(i)}}{b_{2}^{(i)}},
$$

where $a_{2}^{(i)}$ and $b_{2}^{(i)}$ represent the incident and reflected waves at the output of the $i^{\text {th }}$ PA, respectively. The incident wave, $a_{2}^{(i)}$, can be expanded as

$$
a_{2}^{(i)}=\sum_{j=1}^{N} S_{i j} b_{2}^{(j)}
$$

Equation (2) then becomes

$$
\Gamma_{\text {load }}^{(i)}=\frac{\sum_{j=1}^{N} S_{i j} b_{2}^{(j)}}{b_{2}^{(i)}} .
$$

Equation (4) recognizes the reflected and the coupled terms. For simplicity, an assumption is made such that the PA's output is well-matched. This renders the reflected term, $S_{i j} b_{2}^{(j)}, i=j$, negligible. Equation (4) then simplifies to

$$
\Gamma_{\text {load }}^{(i)}=\frac{1}{b_{2}^{(i)}} \sum_{\substack{j=0 \\ j \neq i}}^{N} S_{i j} b_{2}^{(j)} .
$$

Equation (3) also simplifies to

$$
a_{2}^{(i)}=\sum_{\substack{j=0 \\ j \neq i}}^{N} S_{i j} b_{2}^{(j)}
$$

The emulation technique presents a method to generate the time-varying reflection coefficients in (5), while (6) represent the basis of the technique.

\section{Emulation Background}

Due to the time-varying nature of the effected reflection coefficients seen by the PAs, conventional techniques, such as active load-pull, which converges to a constant reflection coefficient is rendered unsuitable as a measurement technique. The solution is then to find a simplified measurement method

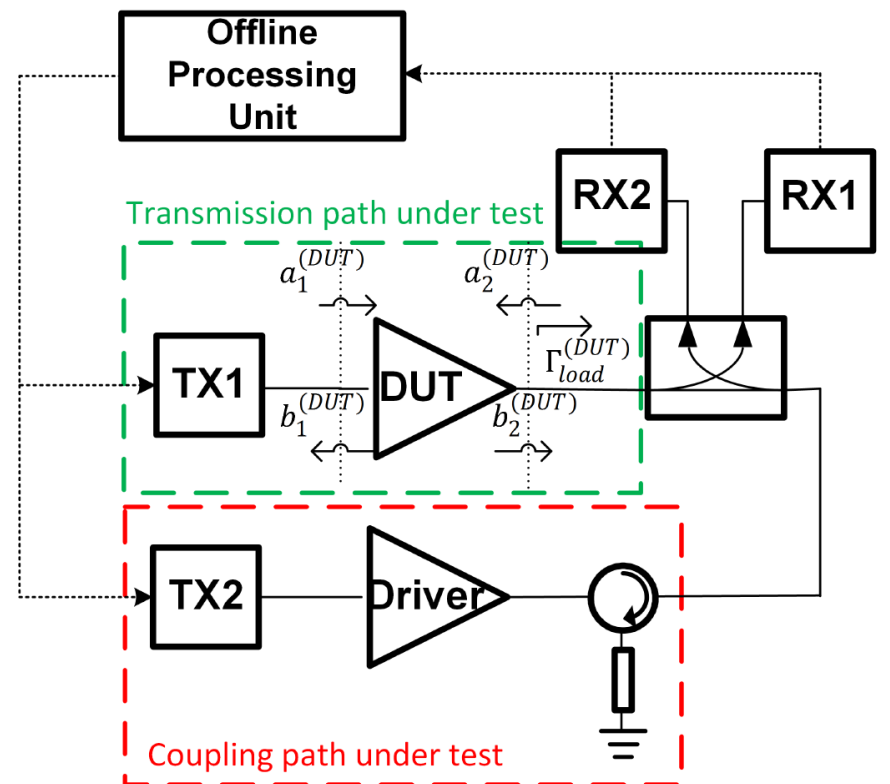

Fig. 3. Measurement setup for the proposed emulation technique.

which can generate such reflection coefficients while converging to a given S-parameter network.

From (5), it can be seen that representing the correct timevarying reflection coefficient, $\Gamma_{\text {load }}^{(i)}$, is a matter of producing accurate representation of all $b_{2}^{(j)}, j \in 1,2, \ldots, N$ in a given antenna array, i.e., the target array. This work presents a unique solution to obtain such representation with a minimal measurement setup requirement by using an iterative method where the interaction between transmission paths are separated in a time-division manner where in each time slot, a transmission path can be represented. The procedure will be discussed in detail in Section III.

\section{EMULATION Method}

As motivated in Section II, the emulation method serves to produce representation of all $b_{2}^{(j)}, j \in 1,2, \ldots, N$ for each PA in order to emulate the effect of time-varying reflection coefficients under a given S-parameter network of the antenna array coupling. The method requires a simple measurement setup to be described. The description here makes further assumption that the PAs in the target antenna array are identical. This assumption can be relaxed through extension of the measurement setup and will be briefly mentioned.

\section{A. Measurement Setup}

The measurement setup is shown in Fig. 3, which consists of

1. An offline processing unit (OPU), e.g., MATLAB

2. Two transmitters (TX1, TX2)

3. A linear amplifier as a driver (independent of the PA in the target array)

4. An isolator

5. A directional coupler

6. Two receivers (RX1, RX2)

7. A PA from the target array, referred to as device under test (DUT). 
TABLE I

ILLUSTRATION OF EMULATION ITERATIVE PROCEDURE:

N ANTENNAS, N TRANSMITTERS, L ITERATIONS

\begin{tabular}{|c|c|c|c|c|}
\hline \multirow{2}{*}{ Iteration } & \multirow{2}{*}{ OPU Time-slot } & \multicolumn{2}{|r|}{ OPU Output } & \multirow{2}{*}{$\begin{array}{c}\text { OPU Input } \\
\text { RX1 } \\
\end{array}$} \\
\hline & & TX1 (TPUT) & TX2 (CPUT) & \\
\hline \multirow[t]{5}{*}{1} & 1 & $a_{1}^{(1)}$ & off & $b_{2}^{(1)}$ \\
\hline & 2 & $a_{1}^{(2)}$ & $S_{21} b_{2}^{(1)}$ & $b_{2}^{(2)}$ \\
\hline & 3 & $a_{1}^{(3)}$ & $S_{31} b_{2}^{(1)}+S_{32} b_{2}^{(2)}$ & $b_{2}^{(3)}$ \\
\hline & $\vdots$ & $\vdots$ & $\vdots$ & $\vdots$ \\
\hline & $\mathrm{N}$ & $a_{1}^{(N)}$ & $S_{N, 1} b_{2}^{(1)}+S_{N, 2} b_{2}^{(2)}+S_{N, 3} b_{2}^{(3)} \ldots+S_{N, N-1} b_{2}^{(N-1)}$ & $b_{2}^{(N)}$ \\
\hline \multirow[t]{5}{*}{2} & 1 & $a_{1}^{(1)}$ & $S_{12} b_{2}^{(2)}+S_{13} b_{2}^{(3)}+\ldots+S_{1, N-1} b_{2}^{(N-1)}+S_{1, N} b_{2}^{(N)}$ & $b_{2}^{(1)}$ \\
\hline & 2 & $a_{1}^{(2)}$ & $S_{21} b_{2}^{(1)}+S_{23} b_{2}^{(3)}+\ldots+S_{2, N-1} b_{2}^{(N-1)}+S_{2, N} b_{2}^{(N)}$ & $b_{2}^{(2)}$ \\
\hline & 3 & $a_{1}^{(3)}$ & $S_{31} b_{2}^{(1)}+S_{32} b_{2}^{(2)}+\ldots+S_{3, N-1} b_{2}^{(N-1)}+S_{3, N} b_{2}^{(N)}$ & $b_{2}^{(3)}$ \\
\hline & $\vdots$ & $\vdots$ & $\vdots$ & $\vdots$ \\
\hline & $\mathrm{N}$ & $a_{1}^{(N)}$ & $S_{N, 1} b_{2}^{(1)}+S_{N, 2} b_{2}^{(2)}+\ldots+S_{N, N-1} b_{2}^{(N-1)}$ & $b_{2}^{(N)}$ \\
\hline$\vdots$ & $\vdots$ & $\vdots$ & $\vdots$ & $\vdots$ \\
\hline \multirow[t]{5}{*}{$\mathrm{L}$} & 1 & $a_{1}^{(1)}$ & $S_{12} b_{2}^{(2)}+S_{13} b_{2}^{(3)}+\ldots+S_{1, N-1} b_{2}^{(N-1)}+S_{1, N} b_{2}^{(N)}$ & $b_{2}^{(1)}$ \\
\hline & 2 & $a_{1}^{(2)}$ & $S_{21} b_{2}^{(1)}+S_{23} b_{2}^{(3)}+\ldots+S_{2, N-1} b_{2}^{(N-1)}+S_{2, N} b_{2}^{(N)}$ & $b_{2}^{(2)}$ \\
\hline & 3 & $a_{1}^{(3)}$ & $S_{31} b_{2}^{(1)}+S_{32} b_{2}^{(2)}+\ldots+S_{3, N-1} b_{2}^{(N-1)}+S_{3, N} b_{2}^{(N)}$ & $b_{2}^{(3)}$ \\
\hline & $\vdots$ & $\vdots$ & $\vdots$ & $\vdots$ \\
\hline & $\mathrm{N}$ & $a_{1}^{(N)}$ & $S_{N, 1} b_{2}^{(1)}+S_{N, 2} b_{2}^{(2)}+\ldots+S_{N, N-1} b_{2}^{(N-1)}$ & $b_{2}^{(N)}$ \\
\hline
\end{tabular}

The notations are with respect to antenna array in Fig. 2. In each iteration, the OPU input overwrites the value from the previous iteration. The system is assumed to be calibrated. TX1 (TPUT) therefore represents $a_{1}^{D U T}$, and TX2 (CPUT) represents $a_{2}^{D U T}$ in Fig. 3.

The DUT and TX1 forms 'transmission path under test' (TPUT), which is used to represent one of the transmission path in the target array. While the driver and TX2 forms 'coupling path under test' (CPUT), representing all the coupled signals from other transmission paths. With the TPUT and CPUT combined, all $b_{2}^{(j)}, j \in 1,2, \ldots, N$ can be represented.

\section{B. Measurement Procedure}

Given that the S-parameter network describing the coupling between the output of all transmission paths is determined and known to the OPU through measurements of an existing design or appropriate simulations, the coupling effect and all $b_{2}^{(j)}, j \in 1,2, \ldots, N$ are emulated through an iterative timedivision procedure, described in Table I. An iteration consists of $N$ OPU time-slots. In an OPU time-slot, the TPUT will be representing one of the transmission paths of the target array. Likewise, the CPUT will represent all the corresponding coupled paths. The received signals at RX1 are then stored at the OPU, before moving to the next OPU time-slot. After the TPUT has represented all the transmission paths in the target array, i.e., after $N$ time-slots, an iteration is complete. The process then repeats itself for a number of iterations, $L$, until the received signals of all TPUTs converge to $N$ timevarying stable solutions. The convergence can be verified by monitoring the error, i.e., the differences between the emulated waveforms from an iteration and the previous iteration.
In the first OPU time-slot of the first iteration, the TPUT will represent the first transmission path in the target array, refer to Fig. 2, by transmitting its intended transmitting signal, $a_{1}^{(1)}$. Since at this point, the coupled signals are not known, CPUT will be off. The received signal of the first transmission path, $b_{2}^{(1)}$, is then stored in the OPU.

In the second OPU time-slot, the TPUT will represent the second transmission path by transmitting $a_{1}^{(2)}$, while CPUT will represent the only known coupled signals $S_{21} b_{2}^{(1)}$. The received signal of the second transmission path, $b_{2}^{(2)}$, is stored in the OPU, before moving on to the next time-slot. The process repeats itself until all $b_{2}^{(j)}, j \in 1,2, \ldots, N$ are known to the OPU.

After the first iteration, the procedure reiterates until each $b_{2}^{(j)}, j \in 1,2, \ldots, N$ converges as described. 


\section{Example Case of Two-element Array with Identical Signal}

The purpose of this sub-section is to provide readers with illustrated example of the emulation method using the most basic application of the method, which is to represent an array with two identical transmission paths (elements), transmitting identical signal, and with symmetric coupling as shown in Fig. 4 , which due to the simplicity of the case foregoes incident and reflected wave notations $\left(a_{1}, b_{2}\right)$ for simpler transmit and receive signals, $x(t)$ and $y(t)$, respectively. The symmetric coupling implies $S_{12}=S_{21}$. Since there are two transmission paths $(N=2)$, which is symmetric and identical, the OPU time-slots definition converges to iterations which further simplifies the case. The iteration develops as follow.

In the first iteration, the coupling signal is unknown and is set to zero. The TX of TPUT transmits the transmitting signal of the target array. The received signal, amplified by the DUT, is stored in the OPU.

In the second iteration, the stored TPUT RX signal from the previous iteration is scaled in amplitude and phase to the corresponding S-parameter network of the target array, and used as the CPUT signal. The TPUT TX signal remains the same due to symmetry. The process then reiterates. After some iterations, in this example four iterations, the coupling signal is fully represented, the TPUT RX signal converges to a single time-varying stable solution, and the procedure is complete. The differences between the TPUT RX signals in the first and the fourth iterations are illustrated through the magnitude spectrum shown in Fig. 5, where it can be observed that the signal in the later iteration develops spectral regrowth and inband distortion, which are expected effects of array coupling. As briefly mentioned, the convergence can be confirmed by monitoring the error between the TPUT RX signal from an iteration and the previous iteration. The error can be quantified, as an example, using normalized mean-square error (NMSE), as shown in Fig. 6.

After the emulation procedure, the received signal can then be analyzed in terms of distortion and impairment, e.g., adjacent channel power ratio, error-vector magnitude, NMSE, and efficiency.

Detailed description and further analysis of this example and other more complicated scenarios are included in Section IV and $\mathrm{V}$.

\section{Isolating Antenna Array Coupling Effects}

The distortion emulated through the described technique is not solely the effect of antenna array coupling, but also the distortion of the PA under $50 \Omega$ termination, which effectively limits the lower level of coupling that can be represented, i.e., reduced measurement dynamic range. This issue can be solved by isolating the coupling effects through digital predistortion of the DUT in the measurement setup. The predistorted signal is then used as the transmitting signal of the target array, i.e., TPUT's TX signal. Multiple work have proposed and described digital predistortion (DPD) techniques [9-12], the emulation procedure described in this paper is not exclusive to any.

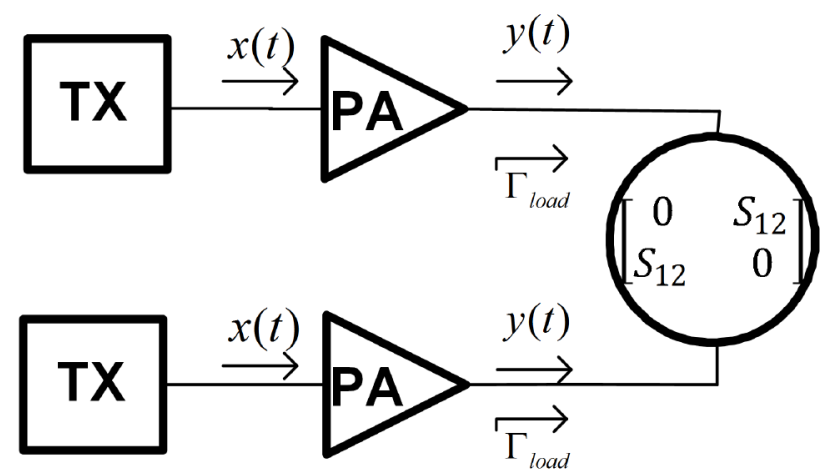

Fig. 4. Two-element array with identical signal and symmetric coupling.

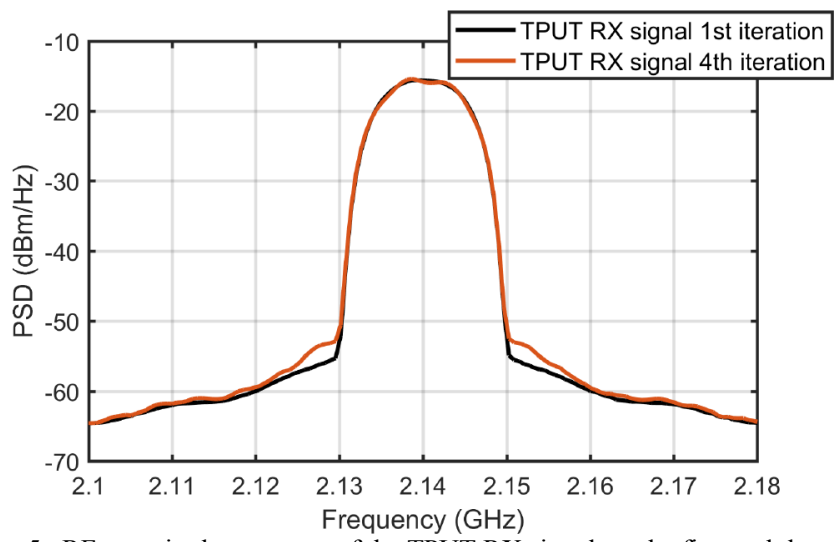

Fig. 5. RF magnitude spectrum of the TPUT RX signals at the first and the fourth iterations.

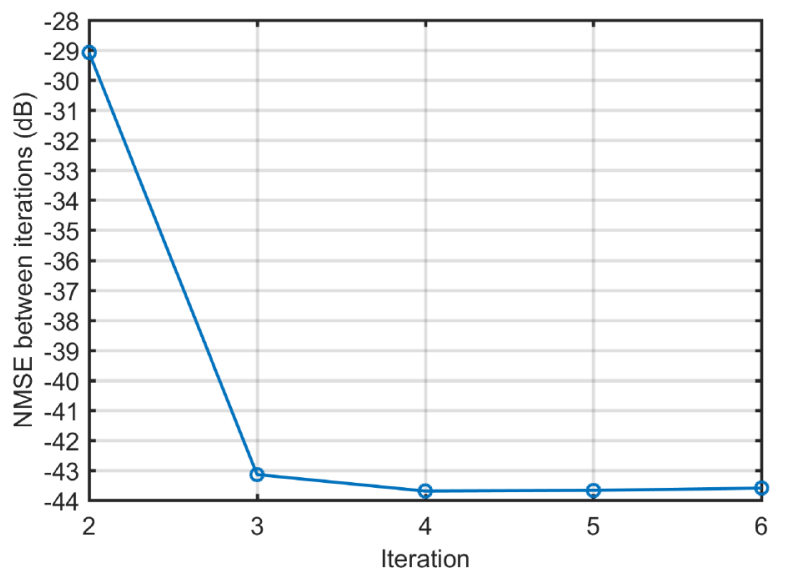

Fig. 6. Error between the TPUT RX signals of an iteration with the previous iteration, quantified through NMSE.

\section{E. Extension to Array with Non-identical Elements}

To extend the method to represent a target array with nonidentical elements is a procedure of adding additional DUTs to the measurement setup, where a group of identical elements requires one DUT. During emulation, TX1 will have to switch between different DUTs to form the corresponding TPUT. The rest of the procedure remains unchanged. 


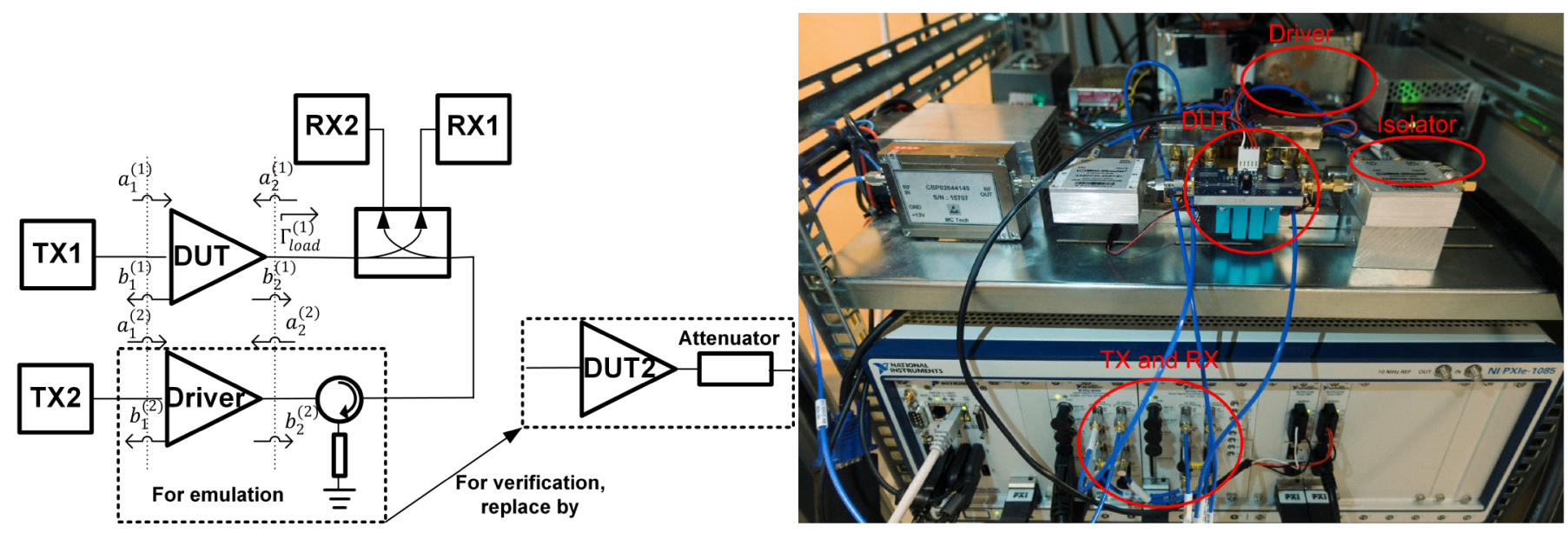

(a)

(b)

Fig. 7. Experimental measurement setup for emulation and verification: (a) Block-diagram modified from [7], and (b) In-lab realization. During verification, output of DUT2 is connected to the output of DUT through an attenuator.

\section{VerificAtion Method of the CASE With TwO- ELEMENT ANTENNA ARRAY}

In order to verify and demonstrate the emulation method of antenna array coupling effects, an experimental measurement setup was built. The setup studies and represents two-element antenna array with identical PAs where each transmission path has its own independent transmitter. A direct comparison can be made between the emulated and the actual coupling effects, for example, in terms of adjacent channel power ratio (ACPR), error-vector magnitude (EVM), and normalized mean square error (NMSE). These quantifiers are interesting as they characterize both in-band and out-of-band distortion.

The setup enables various emulation parameters to be studied including coupling levels, and delay between transmission paths. Additionally, the cross-correlation of the signals in two transmission paths are varied and treated as an important parameter.

\section{A. Transmission Paths Signals' Cross-correlation}

Between the two transmission paths, the signals' crosscorrelation represents different antenna array usage scenarios.

High signals' cross correlation means identical or similar signals are transmitted from the two transmitters. This is representative of a beamforming scenario, where identical signals are transmitted and the delay between transmission paths define the steering angle [13]. In this work, which verifies the two-element array case, the beamforming scenario refers to the cross-correlation coefficient of unity, between the signals in the two transmission paths.

On the other hand, low signals' cross-correlation means independent or dissimilar signals are transmitted in the two transmission paths. This is representative of Massive Multipleinput Multiple-output (MIMO) for multiple users [14]. This scenario is represented with the two transmitting signals' cross-correlation coefficient of 0.0023 in this work.

It is important to note that these two scenarios are two extreme cases. In practice, the signals' cross-correlation can be in between these two extremes.

\section{B. Experimental Measurement Setup}

To demonstrate the proposed measurement technique, the emulation measurement setup shown in Fig. 3 was constructed for demonstration purposes at the Microwave Electronics Laboratory, Chalmers University of Technology as shown in Fig. 7. During the verification procedure, the same setup can be used to represent an actual two-element antenna array simply by replacing the driver and the isolator with another DUT, denoted as DUT2. Coupling S-parameter can then be represented by a two-port device at the output of DUT2. In the verification of this work, coupling is represented by three different attenuators: $16.46,20.55$, and $26.70 \mathrm{~dB}$.

The signals used in emulation and verification are 16-QAM root-raised cosine pulse-shaped, carrying $2^{17}$ bits at $10 \mathrm{MBd}$ or $40 \mathrm{Mbit} / \mathrm{s}$ transmission rate, occupying approximately 10 $\mathrm{MHz} 3-\mathrm{dB}$ bandwidth. The peak-to-average power ratio (PAPR) without DPD is $5.79 \mathrm{~dB}$. To generalize the results, delays between transmission paths are varied from 0 to 2 symbol-period. This normalization of the delay is justified through an additional emulation of the signal at slower symbol-rate of $5 \mathrm{MBd}$.

The transmitters (TX1 and TX2) and receivers (RX1 and RX2) are National Instruments $80 / 200 \mathrm{MHz}$ vector signal transceivers, which combines vector signal generator and analyzer, and field-programmable gate array. The DUTs (DUT1, and DUT2) are Cree's 6W GaN HEMT PAs. RF center frequency of $2.14 \mathrm{GHz}$ was chosen. The driver is different from the DUTs and is a test and measurement amplifier with maximum output power of $47 \mathrm{dBm}$. Full calibration was performed to shift the power and phase reference planes to the DUT. The OPU was implemented in MATLAB. 


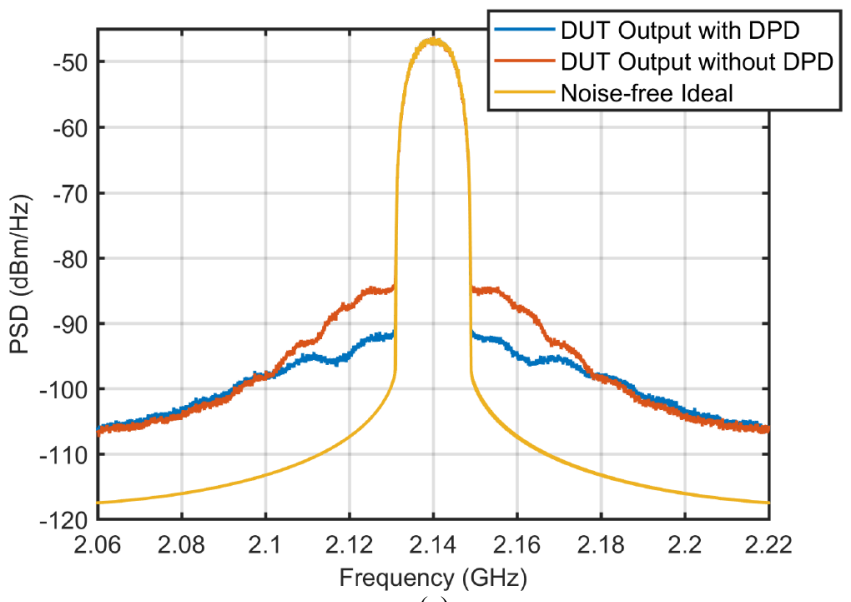

(a)

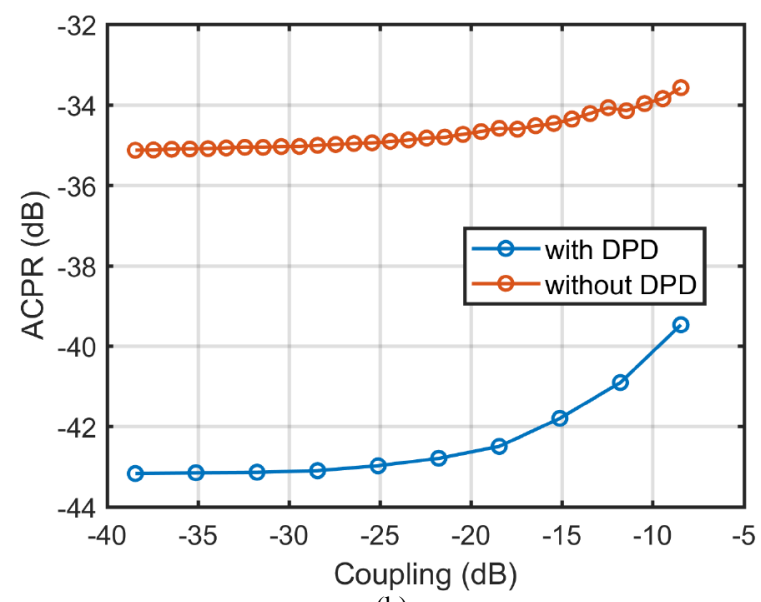

(b)

Fig. 8. Importance of DPD: (a) Magnitude spectrum of the DUT's output with and without DPD, along with noise-free ideal as reference. (b) ACPR vs coupling level with and without DPD.

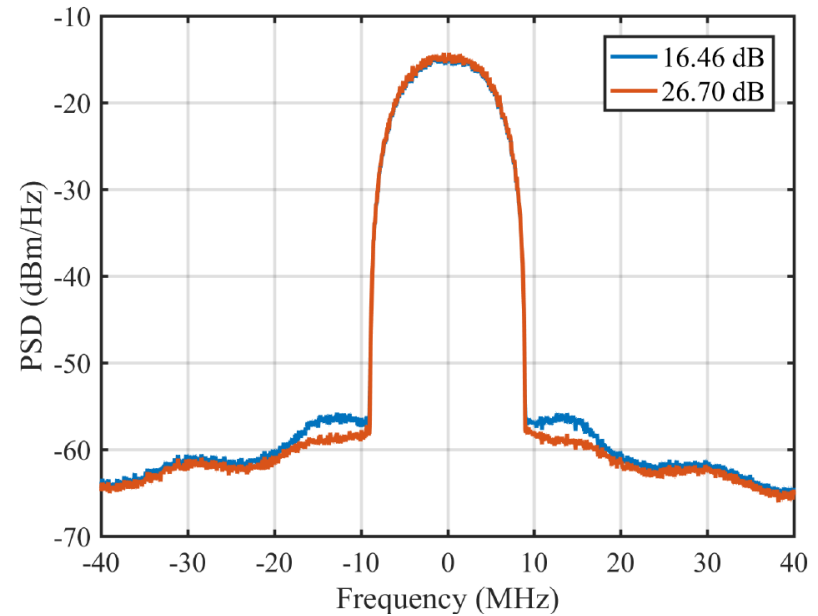

(a)

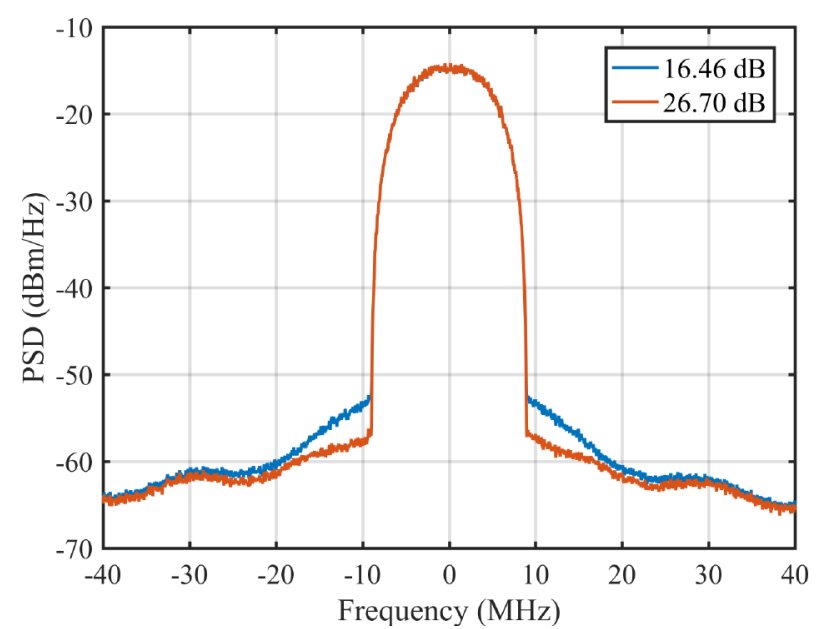

(b)

Fig. 9. Magnitude spectrum of the baseband signals emulated for two-element array. Emulation parameters are zero delay between transmission paths at 16.46 and $26.70 \mathrm{~dB}$ coupling level: (a) Beamforming scenario, and (b) Massive MIMO scenario.

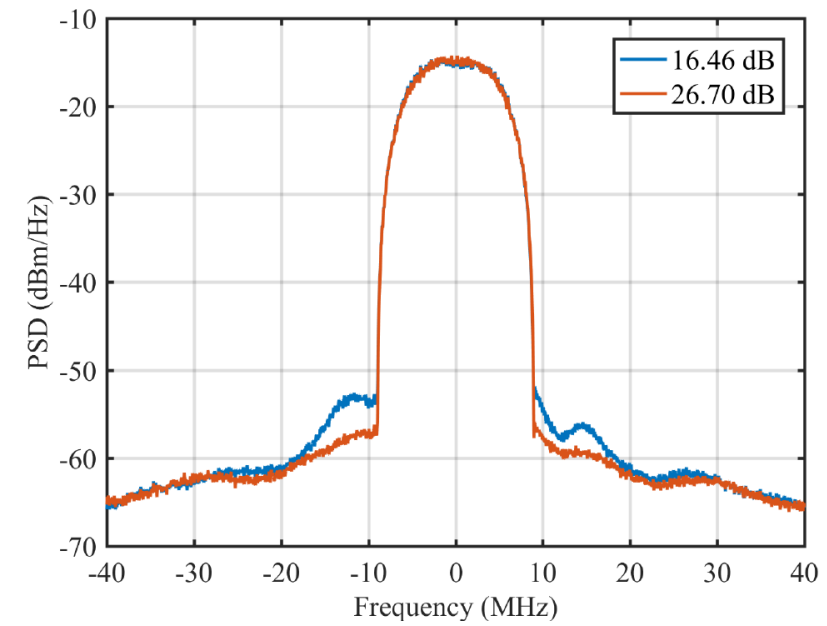

(a)

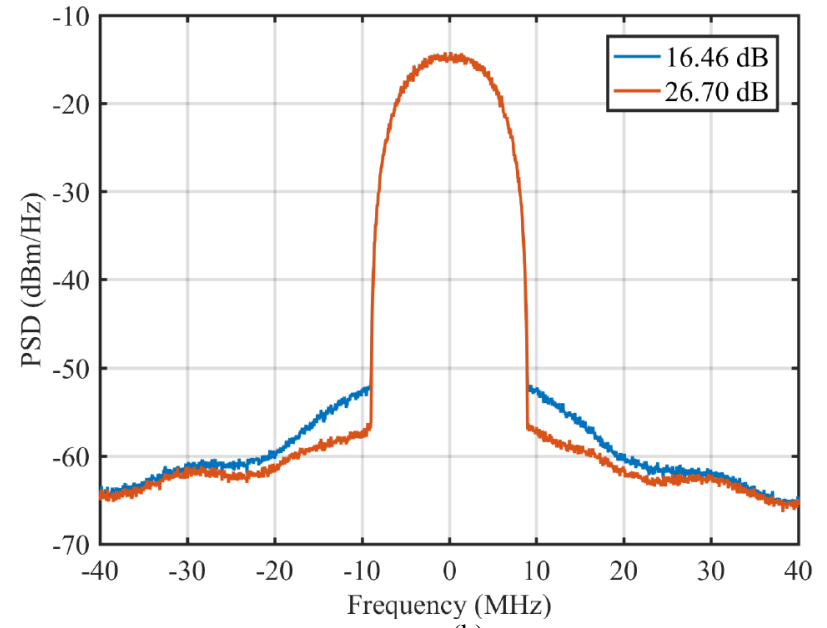

(b)

Fig. 10. Magnitude spectrum of the baseband signals emulated for two-element array. Emulation parameters are 0.5 symbol-period delay between transmission paths at 16.46 and $26.70 \mathrm{~dB}$ coupling level: (a) Beamforming scenario, and (b) Massive MIMO scenario. 


\section{Digital Predistortion}

As mentioned in Section III, the distortion of the DUT under $50 \Omega$ output termination, can be compensated by performing digital predistortion (DPD). The DPD technique used to identify the pre-distorter parameters in this work is a variant of indirect learning architecture [9] as described in [15]. In the scope of this work, the pre-distorter parameters are extracted while the DUT is terminated with $50 \Omega$.

Terminating the DUT with $50 \Omega$, the nonlinear distortion shown through a test signal was quantified as NMSE of -27.9 $\mathrm{dB}$, and ACPR of $-35.3 \mathrm{dBc}$. With DPD, the NMSE reduced to $-37.2 \mathrm{~dB}$, while the ACPR reduced to $-43.8 \mathrm{~dB}$. The improvement is also shown through magnitude spectrum of the DUT's output with and without DPD in Fig. 8(a), where it can be observed that the spectral regrowth without DPD is more pronounced.

It was also mentioned in Section III that the distortion of the DUT under $50 \Omega$ termination, if not taken care of, results in reducing the dynamic range of the emulation measurement. This effect can be seen when observing emulated ACPR as a function of coupling level of 8.5 to $38.5 \mathrm{~dB}$ in the target array, shown in Fig. 8(b). It can be seen that the emulated ACPR with DPD spans from $-39.5 \mathrm{~dB}$ at the highest coupling level and reduce to the noise-limited of $-43.2 \mathrm{~dB}$ at the lowest coupling level, effectively spanning ACPR range of $3.7 \mathrm{~dB}$. On the other hand, without DPD the emulated ACPR range is merely $1.6 \mathrm{~dB}$, since the emulated result is dominated by the distortion of the DUT relatively close-to $50 \Omega$ termination at lower coupling level instead of the coupling effect.

\section{RESUlTS OF Two-ELEMENT ANTENNA ARRAY EMULATION AND VERIFICATION}

This section presents the results of the emulation of twoelement array as described in Section IV. For verification of the emulation technique, verification is performed by comparison of the emulated signals with the signals from the target array. As discussed, parameters of interest are coupling level, delay between transmission paths, and array usage scenario.

\section{A. Emulated Spectrum}

The magnitude spectrum of the emulated waveforms are shown in Fig. 9 and Fig. 10. In all figures, the results from emulation of an array with 16.46 and $26.70 \mathrm{~dB}$ coupling levels are shown. Beamforming and Massive MIMO scenarios are shown in (a) and (b) of the two figures, respectively. In Fig. 9, the emulation targets zero-delay between transmission paths, while in Fig. 10, the emulation targets 0.5 symbol-period delay.

Observing the zero-delay case in Fig. 9, it can be seen that under the same coupling level, spectral regrowth is more pronounced in Massive MIMO scenario. In contrast, observing the 0.5 symbol-period emulated spectrum in Fig. 10 reveals that spectral regrowth are pronounced under both beamforming and Massive MIMO scenarios. These findings imply that delay between transmission paths are justified as an important parameter to emulate under beamforming scenario. While under Massive MIMO scenario, spectral regrowth are pronounced in both delay cases, implying that in such scenario, delay is less of an important parameter. This will be further discussed through ACPR, EVM, and NMSE.

\section{B. Verification with Adjacent Channel Power Ratio, Error- vector Magnitude, and Normalized Mean Square Error}

Fig. 11 and Fig. 12 show the results of both the emulation and the verification setup in terms of ACPR, EVM, and NMSE as functions of the normalized delay. Fig. 11 shows these results under beamforming scenario from 0 to 2 symbolperiod. Fig. 12 shows the results under Massive MIMO from 0 to 1 symbol-period.

Comparing the ACPR, EVM, and NMSE from emulation with the ones from verification in both beamforming and Massive MIMO scenarios show good agreement in the three quantifiers in all studied coupling levels and delay span. The emulation measurement technique is therefore verified through experiment.

To validate delay normalization, in Fig. 11, at $16.46 \mathrm{~dB}$ coupling level, comparing the results from $10 \mathrm{MBd}(40$ $\mathrm{Mbit} / \mathrm{s})$ transmission rate with the ones from $5 \mathrm{MBd}(20$ Mbit/s) transmission rate also show good agreement in all three quantifiers throughout the delay span. This justifies the generalization of results with regards to delay normalization, proving the technique useful for both low and high transmission rates.

Similarly to the emulated spectrums shown in Fig. 9 and Fig. 10, it can be observed from the three quantifiers that the beamforming scenario is sensitive to the delay relative to the Massive MIMO scenario. Specifically at $16.46 \mathrm{~dB}$ coupling level, under beamforming scenario, the emulated and verified ACPR, shown in Fig. 11(a), spans from approximately -41 to $39 \mathrm{dBc}$. In contrast, under the same coupling level, but under Massive MIMO scenario, the ACPR, shown in Fig. 12(a), spans from -40.5 to $-39.5 \mathrm{dBc}$. Furthermore, observing the EVM at the same coupling level, the beamforming results in Fig. 11(b) show a span from 1 to $4.5 \%$. However, for Massive MIMO in Fig. 12(b), the span is reduced to 3.4 to $4.1 \%$. NMSE results at the same coupling level further support these results. In Fig. 11(c) the beamforming NMSE spans from -34 to $-26 \mathrm{~dB}$. While the results from Massive MIMO in Fig. 12(c) reveals almost constant value of $-26 \mathrm{~dB}$. These findings are not surprising, because in the beamforming scenario, the signals in the two transmission paths become less correlated in time as the delay increases, while for Massive MIMO, the signals are uncorrelated regardless of the delay between transmission paths.

Even though the emulation and the verification show good agreement especially in terms of the resulting trends, slight discrepancies are observed. The discussions on these discrepancies and their causes continue in Section VIII. 


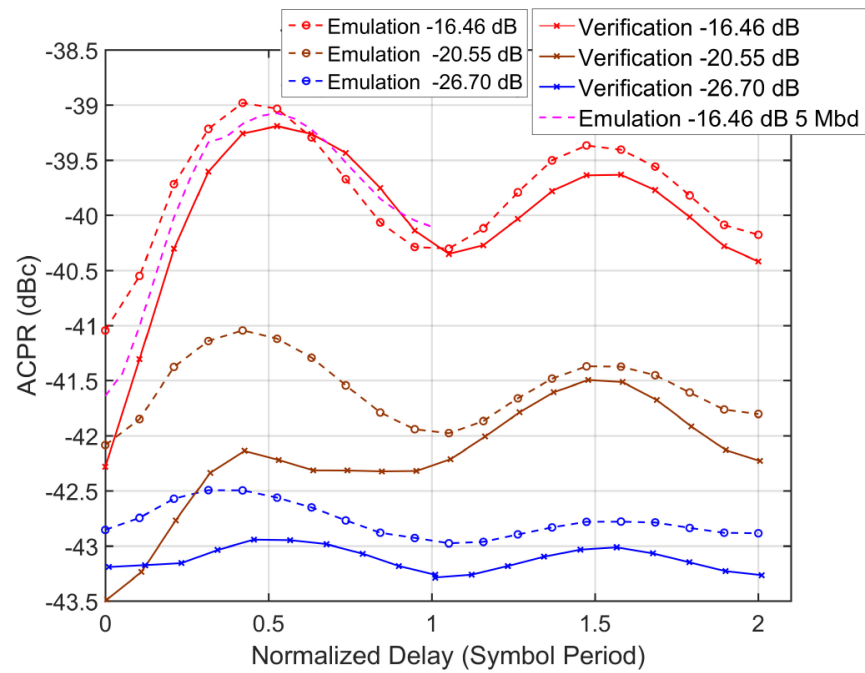

(a)

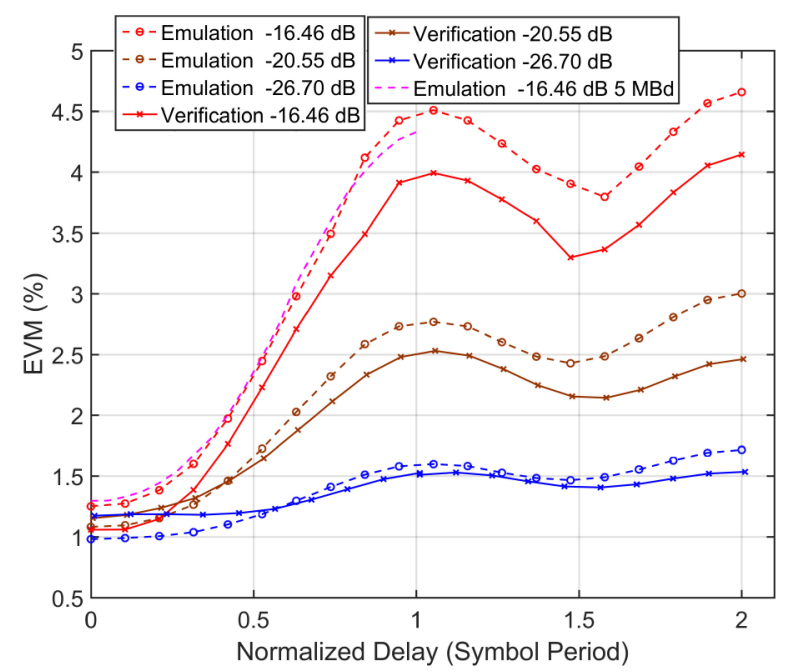

(b)

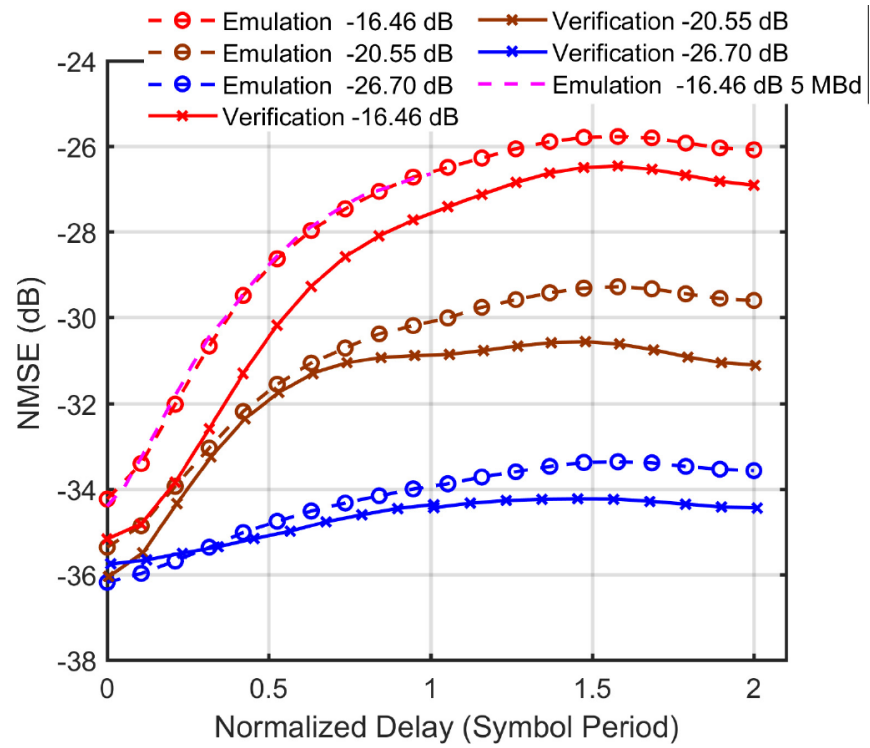

(c)

Fig. 11. Beamforming scenario emulation and verification results vs delay: (a) ACPR from [7], (b) EVM from [7], and (c) NMSE.

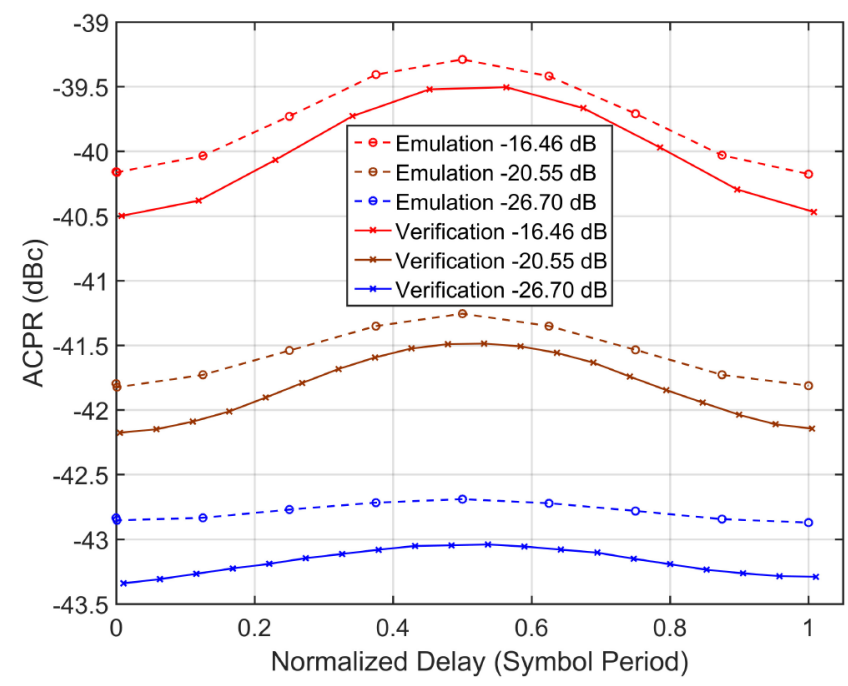

(a)

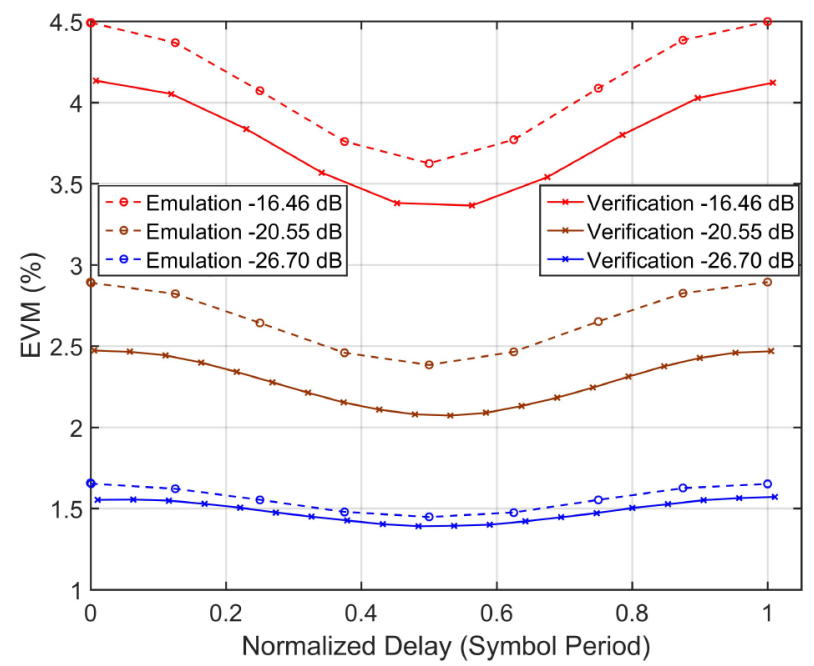

(b)

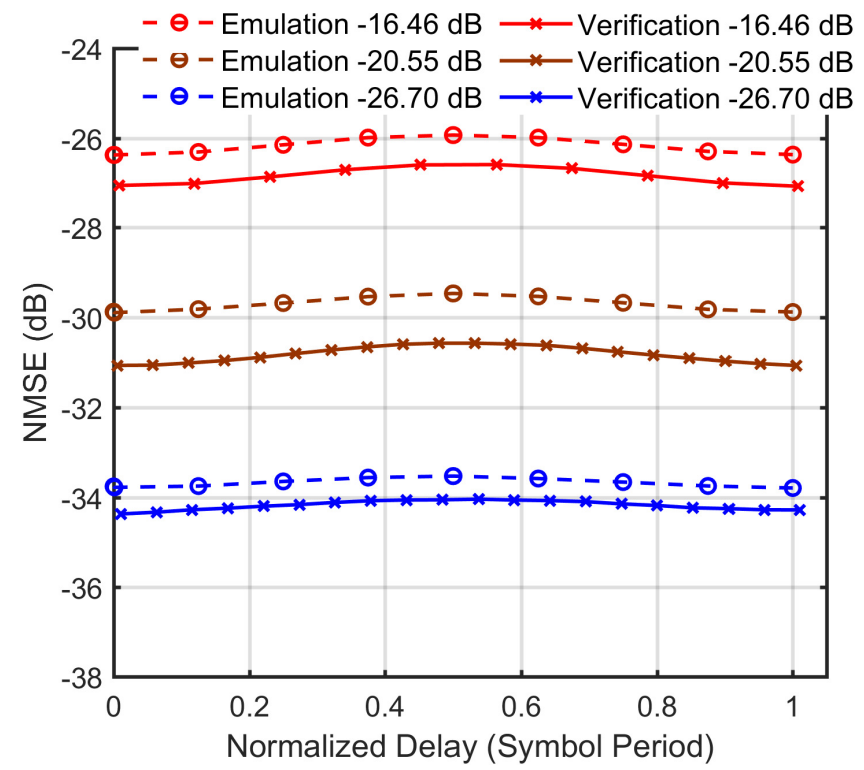

(c)

Fig. 12. Massive MIMO scenario emulation and verification results vs delay: (a) ACPR from [7], (b) EVM from [7], and (c) NMSE. 


\section{COMPARISON WITH CONVENTIONAL LOAD-PULL}

Conventional load-pull techniques start with a known target reflection coefficient [8]. However, in cases where two amplifiers are coupled, the reflection coefficient is a function of the coupling factor, as well as the two output signals themselves including their distortion. Thus, the reflection coefficient is not a priori known, which precludes the use of convention load-pull systems to study such cases. On the other hand, the proposed emulation method is able to present the correct time-varying reflection coefficient to the corresponding DUT. This ability distinguishes the proposed technique from the well-known active load-pull techniques.

To help illustrate the difficulty of realizing the correct reflection coefficient through active load-pull under output coupling, Fig. 13 and Fig. 14 show the magnitude of the emulated reflection coefficient, $\tilde{\Gamma}_{\text {Load }}$, as a function of frequency offset from the center frequency of $2.14 \mathrm{GHz}$, which is obtained from the setup described in Section IV. Fig. 13 shows $\tilde{\Gamma}_{\text {Load }}$ under beamforming scenario, while Fig. 14 shows $\tilde{\Gamma}_{\text {Load }}$ under Massive MIMO scenario. The case with no delay between transmission paths are shown in (a) while maximum studied delay is shown in (b). Note that the reflection coefficient as a function of frequency shown in Fig. 14 is deterministic, and not noise as may be perceived at first glance.

Observing Fig. 13(a), it can be seen that $\tilde{\Gamma}_{\text {Load }}$ shows a roughly linear magnitude profile across the in-band frequency range. These findings imply that beamforming with no-delay between transmission paths is a special case, which can be emulated with conventional load-pull technique, since the target reflection coefficient is equal to aiming for the target coupling level when matched and reciprocal. Nevertheless, it is worth mentioning that with unmatched or non-reciprocal coupling the reflection coefficient is altered. On the other hand, observing $\tilde{\Gamma}_{\text {Load }}$ shown in the case with delay in Fig. 13(b), reveals that to obtain such reflection coefficient through active load-pull would entail further complications, namely, the time-varying frequency dependent target reflection coefficients are unknown a priori. Furthermore, observing Fig. 14, the Massive MIMO scenarios show rapidly fluctuating magnitude and phase. This is as expected as the output signal, $b_{2}$, and the coupling signal, $a_{2}$, are uncorrelated, regardless of the delay, resulting in time-varying and highly frequency dependent reflection coefficients, which are unknown a priori. This implicates that a conventional load pull technique may not be able to emulate such scenario.

To support and conclude this comparison, Fig. 15 shows the measured reflection coefficient created with a conventional active load-pull system. The target reflection coefficient of the active load-pull is from $\tilde{\Gamma}_{\text {Load }}$ in Fig. 13(a), i.e., beamforming scenario without delay. Constraining to the cases without delay, the three sets of output signals are subsequently compared in terms of the emulated distortion, quantified through ACPR, EVM, and NMSE, as given in Table II. It can be seen through these quantifiers that conventional active load-pull provides distortion somewhat comparable to the emulation of beamforming scenario since it is a special case as discussed. The same, however, cannot be said about the Massive MIMO scenario even at the same coupling, since the target time-varying and frequency dependent reflection coefficients are unknown a priori.

\section{EMULATION AND VERIFICATION OF THREE-ELEMENT ANTENNA ARRAY}

To further verify the emulation technique, comparisons between emulation and verification representing three-element antenna array is provided. The case under consideration is one transmitter transmitting through three transmission paths affected by output coupling.

A verification setup is built to represent a three-element antenna array, as shown in Fig. 16. The three DUTs (DUT1, DUT2, and DUT3), which are the GaN HEMT PAs described in Section IV, are fed using one transmitter (TX1) through input power splitting network. The minimum isolation between the DUTs input is $24.17 \mathrm{~dB}$ at the center frequency of $2.14 \mathrm{GHz}$. A 6-dB splitter, terminated on all three ports with attenuators, is used to represent coupling network at the output paths of the three DUTs. The values of the attenuators are uniformly varied with values of 3,6 , and $10 \mathrm{~dB}$. This results in represented coupling level between each path of approximately 12,18 , and $26 \mathrm{~dB}$, respectively.

The emulation setup remains the same as described in Section IV. De-embedding was performed to take into account the attenuation and delay of the input network. The coupling network is measured for S-parameter using a vector network analyzer, resulting in a $3 \times 3 \mathrm{~S}$-parameter matrix for each set of attenuators.

The test signal remains the same as described in Section IV. The emulation and the verification are performed with and without DPD, where the DPD nonlinear coefficients are extracted from DUT1.

The emulated and the verified output waveforms of DUT1, $b_{2}^{(1)}$, are compared in Fig. 17, quantified through ACPR and EVM, which show that both the out-of-band and the in-band distortion increases as coupling level increase. Finally, the results from the case with DPD show good agreement, while the case without DPD shows a slight shift between emulation and the verification. The causes of the discrepancy are further discussed in Section VIII. 


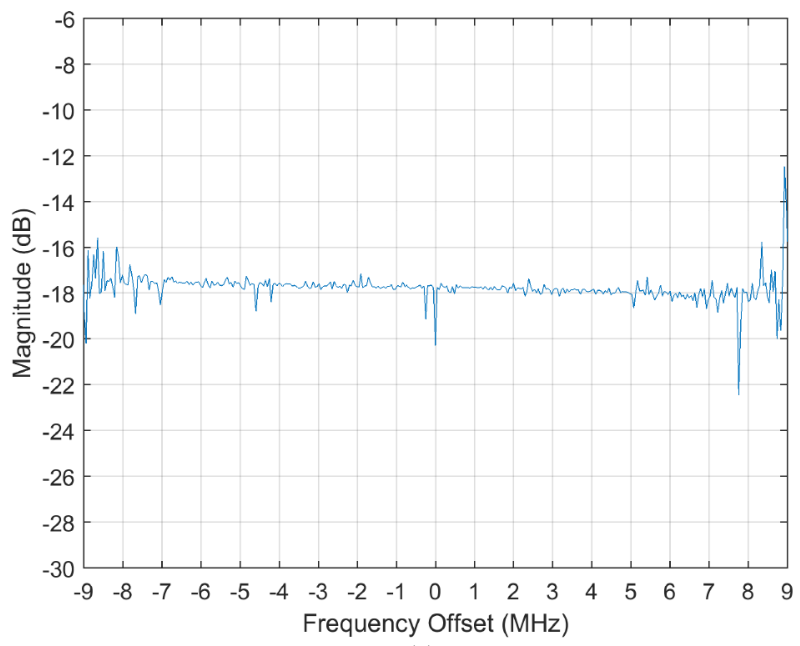

(a)

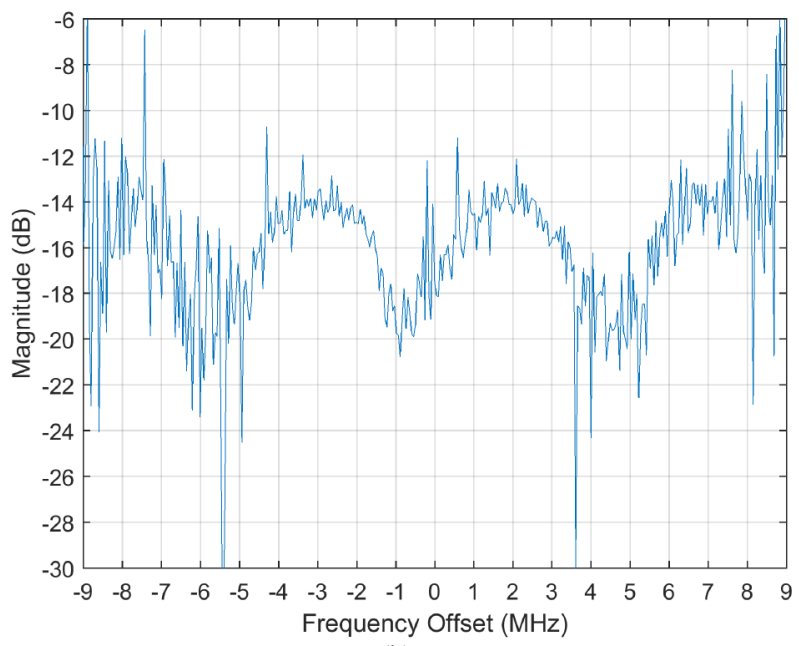

(b)

Fig. 13. Magnitude of the emulated reflection coefficient under beamforming scenario with $16.46 \mathrm{~dB}$ coupling, delay between transmission paths: (a) nodelay, and (b) 2 symbol-period delay.

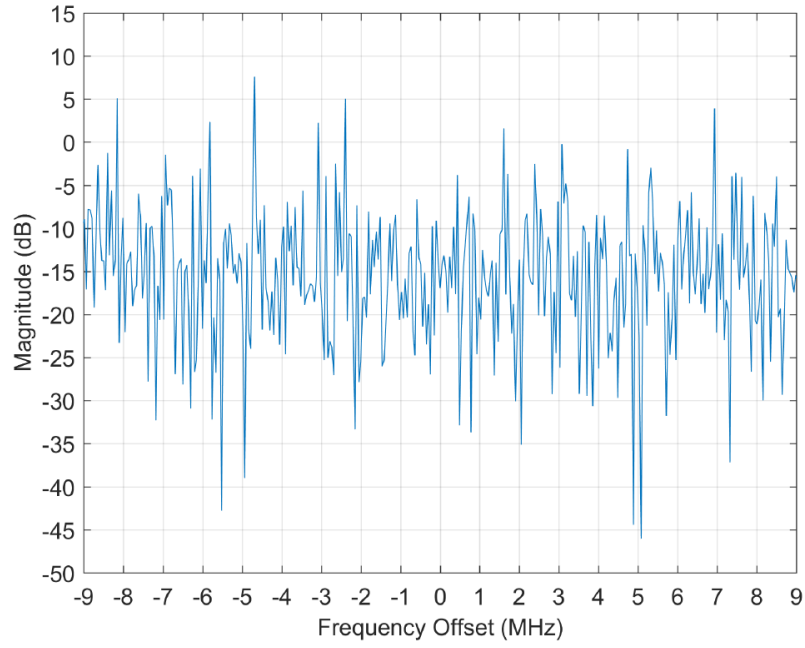

(a)

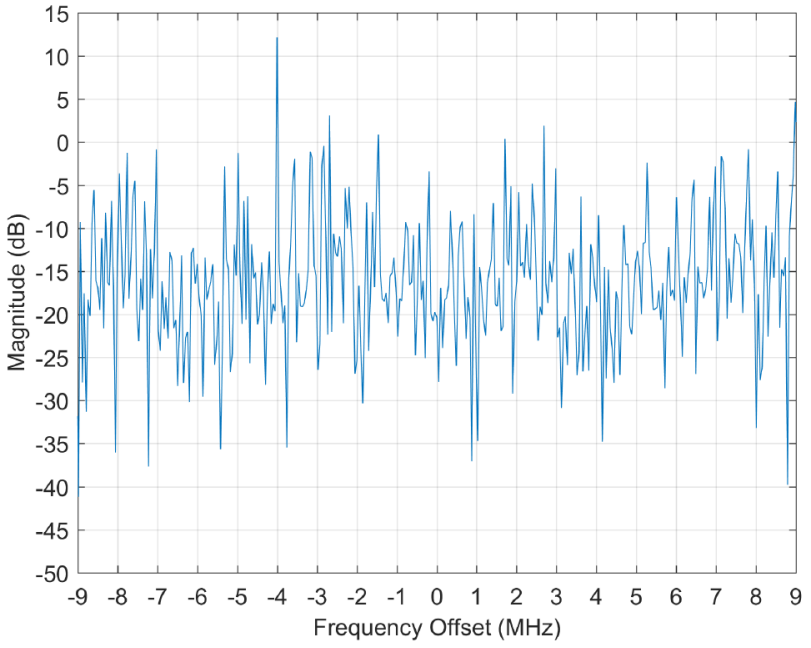

(b)

Fig. 14. Magnitude of the emulated reflection coefficient under Massive MIMO scenario with $16.46 \mathrm{~dB}$ coupling, delay between transmission paths: (a) nodelay, and (b) 1 symbol-period delay.

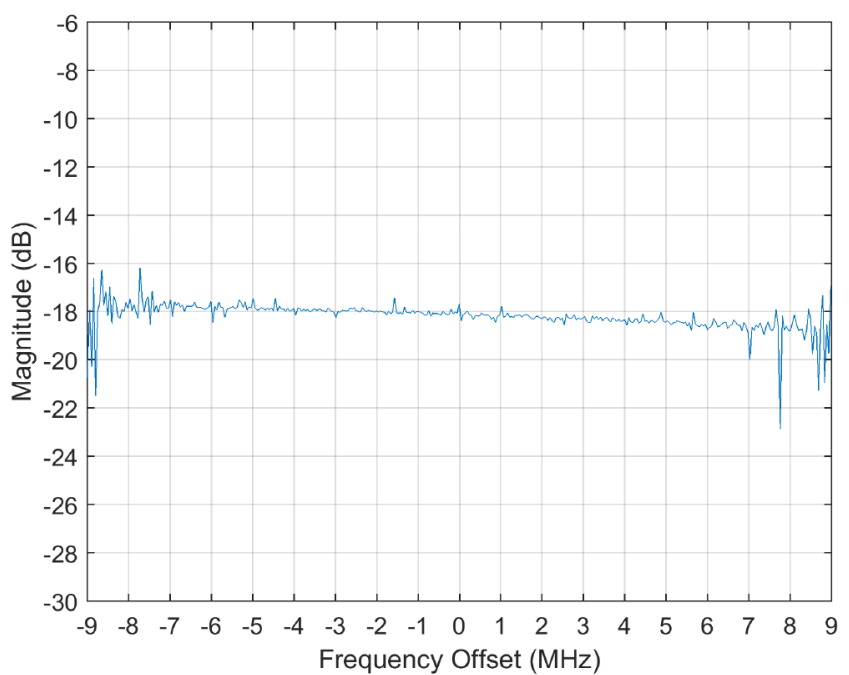

TABLE II

CONVENTIONAL ACTIVE LOAD-PULL VS EMULATION

\begin{tabular}{cccc}
\hline \hline Results & $\begin{array}{c}\text { Active Load- } \\
\text { pull }\end{array}$ & Beamforming & $\begin{array}{c}\text { Massive } \\
\text { MIMO }\end{array}$ \\
\hline ACPR (dBc) & -41.49 & -41.04 & -40.16 \\
EVM (\%) & 1.36 & 1.25 & 4.491 \\
NMSE (dB) & -34.05 & -34.23 & -26.37 \\
\hline \hline
\end{tabular}

Target array: Coupling level $16.46 \mathrm{~dB}$, no-delay between transmission paths.

Fig. 15. Magnitude of the active load-pull reflection coefficient under beamforming scenario with $16.46 \mathrm{~dB}$ coupling and no-delay between transmission paths. The target reflection coefficient is obtained from Fig. 13(a). 


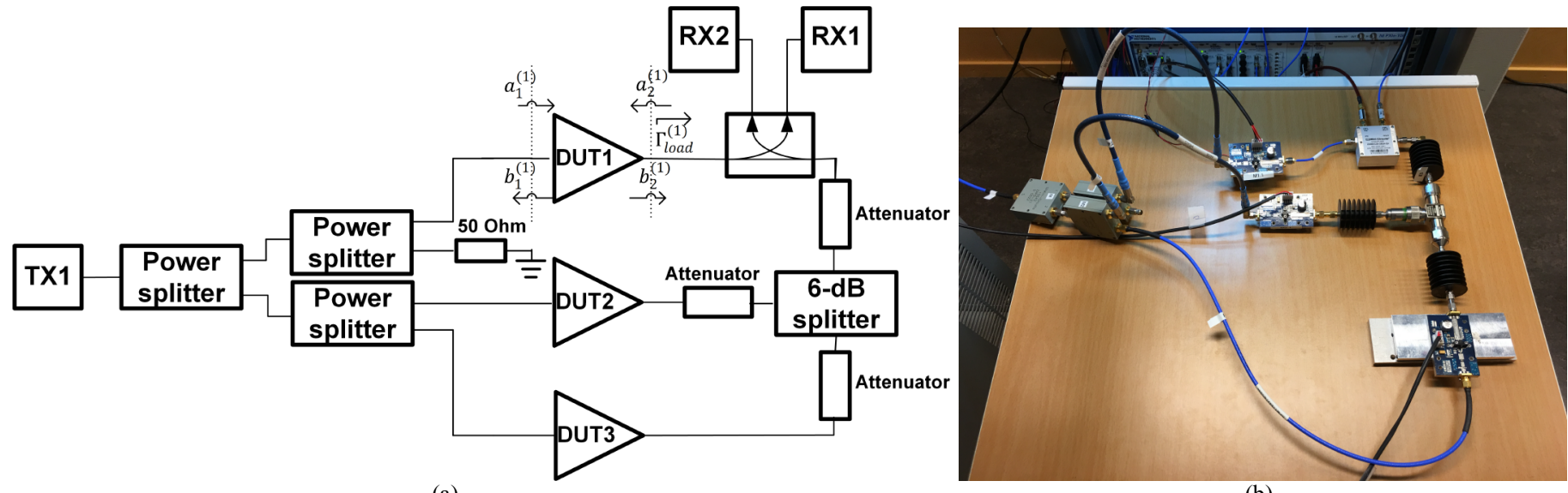

(a)

(b)

Fig. 16. Experimental setup for verification of three-element array: (a) block-diagram, and (b) in-lab realization.

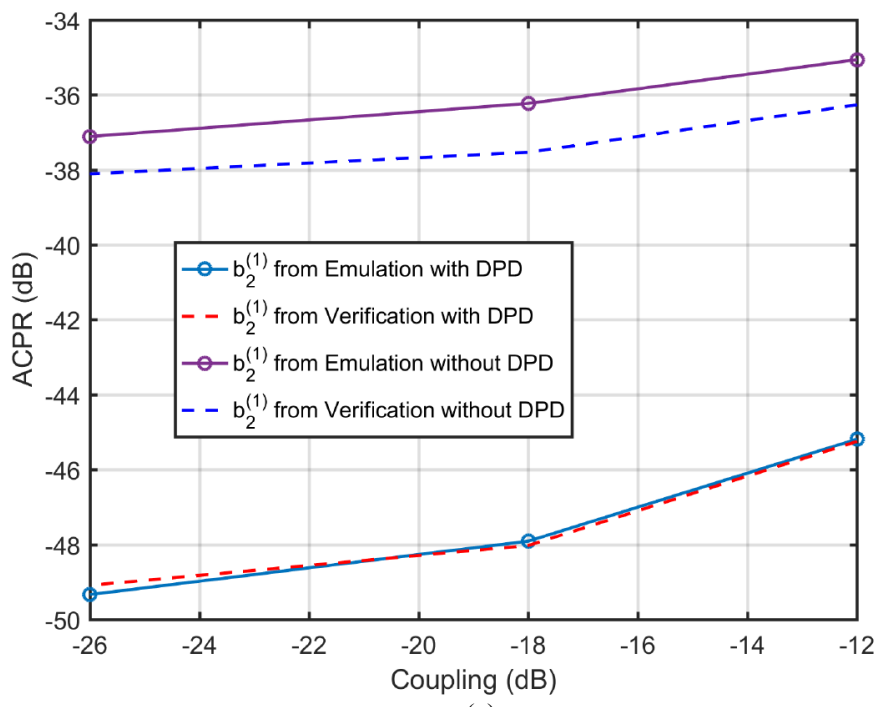

(a)

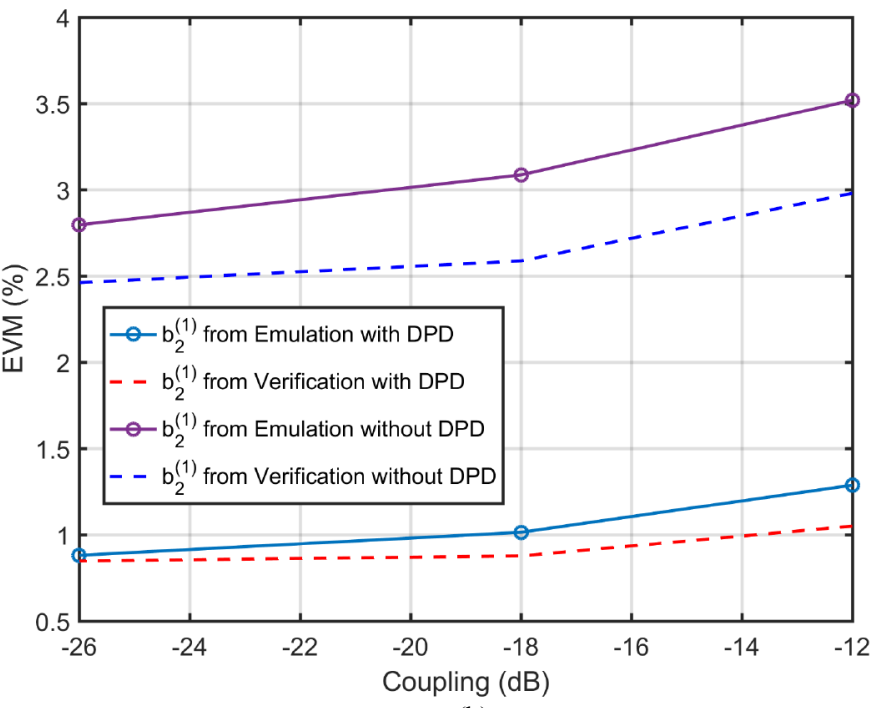

(b)

Fig. 17. Three-element array emulation and verification results: (a) ACPR, and (b) EVM.

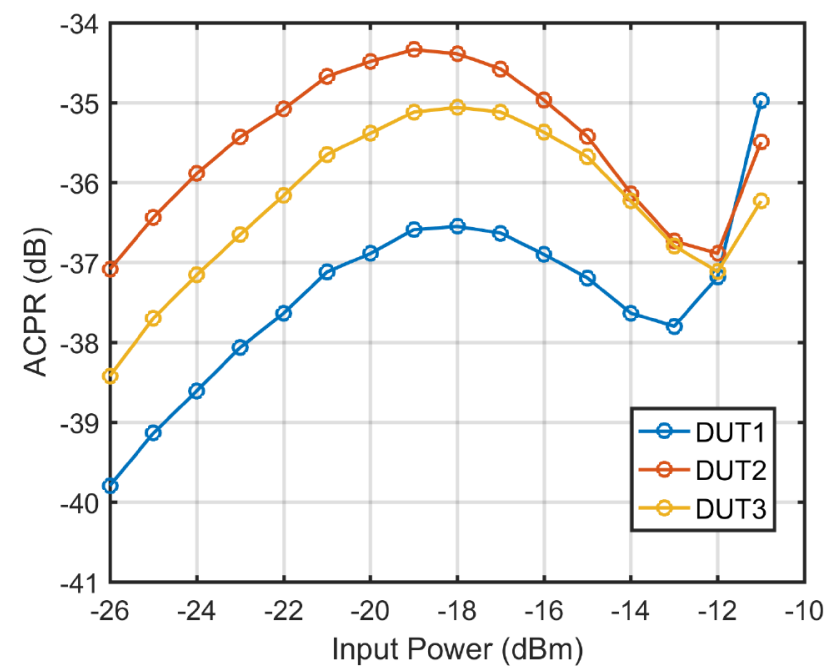

(a)

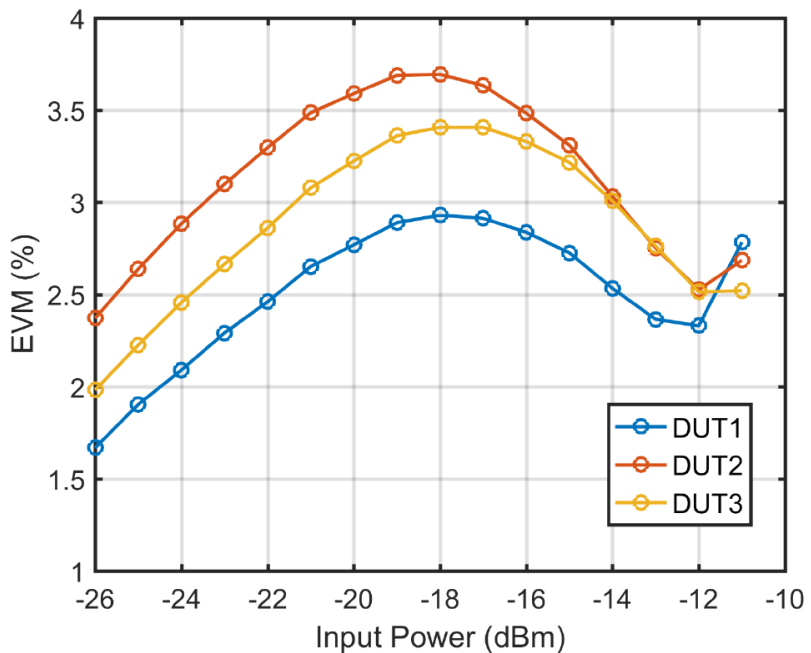

(b)

Fig. 18. Discrepancies between each PA used in the emulation and the verification, quantified through: (a) ACPR, and (b) EVM. 


\section{DISCUSSIONS ON DISCREPANCY BETWEEN EMULATION AND VERIFICATION}

Observing the results from the emulation and the verification of arrays with two and three elements in Section $\mathrm{V}$ and Section VII, respectively, shows overall good agreement, with slight discrepancies varying from scenario to scenario. Even though these discrepancies are marginal and mainly affect the absolute values instead of the relative difference from one scenario to another, i.e., the trends of the results, their causes deserve some discussions to further improve the technique in the future and to provide descriptions of pitfalls users of the technique may wish to avoid depending on the level of accuracy that is needed. These discrepancies are mainly attributed from two imperfections not taken into account in the emulation setup, namely, non-identical elements, and uncontrolled higher harmonics.

The assumption on identical elements validates the use of only one PA as DUT as discussed in Section III. However, the 6W GaN HEMT PAs used in these experiments as DUT1, DUT2, and DUT3 are not ideally identical. This is exhibited through the varying level of distortion at $50 \Omega$ termination, quantified through ACPR and EVM as functions of input power level, shown in Fig. 18. Note that DUT1 and DUT2 are used in the two-element array verification in Section IV and V. DUT1, DUT2, and DUT3 are used in the three-element array verification in Section VII. DUT1 is used in all emulation as the DUT.

Furthermore, the higher-order harmonics of the DUT output signal affect the intermodulation products, which in turn affect the quantified distortion. Due to the relatively narrow-band nature of the isolator used in the emulation setup in this work, the reflection of the higher harmonics is uncontrolled. Nevertheless, such effects observed in this work is marginal due to the harmonic terminations of the $6 \mathrm{~W}$ GaN HEMT PAs that were used.

\section{CONCLUSION}

A measurement technique to emulate coupling effects between the output of power amplifiers has been proposed. The technique has been theoretically motivated. The full procedure was described. The technique is experimentally demonstrated and verified under various coupling levels, delays between transmission paths, usage scenarios, and number of elements in the target array. Pitfalls of the technique are also discussed which serve to provide users of the technique with information on points to consider, depending on the desired level of accuracy. Comparisons with conventional active load-pull are also provided. The technique is suitable for studying the distortion generated due to coupling effects at the output of power amplifiers such as that in an antenna array without the need for construction of the actual array or coupling network. The technique also requires minimal number of power amplifiers as device under tests, where a group of identical power amplifiers can be represented using one device under test. The technique is therefore suitable for obtaining a quick experimental verification of the design-goal.
The emulation method can also be used beyond what was demonstrated in this paper and instead of antenna array, can be applied to multi-port power amplifier architectures, such as Doherty [16] or out-phasing [17] amplifiers. Deeper insight can be obtained about the waveforms of the individual amplifier, before the power combiner, which is typically not easy to realize in a measurement setup, except with great difficulty [18]. Due to the versatility of the measurement technique, it can provide designers of future communication systems with a tool for fast investigation into their design trade-offs and compromises.

\section{ACKNOWLEDGEMENT}

The authors would like to express their gratitude towards the following: K. Hausmair and Prof. T. Eriksson, both at the Chalmers University of Technology (Chalmers), for fruitful discussions on digital pre-distortion; V. Tokmakis at Microwave Electronics Laboratory (MEL), Chalmers, for hardware and software support; S. Gustafsson at MEL for his assistance; Prof. H. Zirath at MEL for fruitful discussions. P. Tehrani, L. Johansson, and T. Inoue, all from National Instruments.

\section{REFERENCES}

[1] L. C. Godara, "Application of antenna arrays to mobile communications, part II: beam-forming and direction-of-arrival considerations," Proc. IEEE, vol. 85, no. 8, pp. 1195-1245, Aug. 1997.

[2] E. G. Larsson, O. Edfors, and F. Tufvesson, "Massive MIMO for next generation wireless systems," IEEE Commun Mag., vol. 20, no. 2, pp. 186-195, Feb. 2014.

[3] Y. J. Cheng, "Millimeter-wave high-gain wideband planar integrated antenna array," in IEEE International Conference on Communication Problem-Solving, Beijing, 2014, pp. 675-676.

[4] C. Fager, X. Bland, K. Hausmair, J. Chani Cahuana, and T. Eriksson, "Prediction of smart antenna transmitter characteristics using a new behavioral modeling approach," in 2014 IEEE MTT-S International Microwave Symposium (IMS 2014), Tampa, FL, 2014, pp. 1-4.

[5] K. Hausmair, S. Gustafsson, C. S. Sanchez-Perez, P. N. Landin, U. Gustavsson, T. Eriksson, and C. Fager, "Prediction of nonlinear distortion in wideband active antenna arrays," IEEE Trans. Microw. Theory Tech., vol. PP, no. 99, pp 1-14.

[6] D. Nopchinda, and K. Buisman, "Emulation of array coupling influence on RF power amplifiers in a measurement setup," in Microwave Measurement Conference (ARFTG), San Francisco, 2016, pp. 1-4.

[7] D. Nopchinda, and K. Buisman, "An active load-pull technique creating time-variant impedances to emulate coupling between power amplifiers," in 2017 IEEE MTT-S International Microwave Symposium (IMS 2017), Honolulu, 2017, pp. 1-4.

[8] M. Marchetti, M. J. Pelk, K. Buisman, W. C. E. Neo, M. Spirito and L. C. N. de Vreede, "Active harmonic load-pull with realistic wideband communications signals," IEEE Trans. Microw. Theory Tech., vol. 56, no. 12 , pp. 2979-2988, Dec. 2008.

[9] C. Eun, and E. Powers, "A new Volterra predistorter based on the indirect learning architecture," IEEE Trans. Signal Process., vol. 45, no. 1, pp. 223-227, Jan. 1997.

[10] L. Ding, G. T. Zhou, D. R. Morgan, Z. Ma, J. S. Kenney, J. Kim, and C. R. Giardina, "A robust digital baseband predistorter constructed using memory polynomials," IEEE Trans Commun., vol. 52, no. 1, pp. 159165, Jan. 2004.

[11] D. R. Morgan, Z. Ma, J. Kim, M. G. Zierdt, and J. Pastalan, "A generalized memory polynomial model for digital predistortion of RF power amplifiers," IEEE Trans. Signal Process., vol. 54, no. 10, pp. 3852-3860, Oct. 2006.

[12] J. Kim, and K. Konstantinou, "Digital predistortion of wideband signals based on power amplifier model with memory," Electron. Lett., vol. 37, no. 23, pp. 1417-1418, Nov. 2001. 
[13] R. Benjamin, "On the use of delays for wide-band superdirective beam forming in endfire and broadside arrays," Radio and Electronic Engineer, vol. 45, no. 9, pp. 451-461, Sep. 1975.

[14] X. Gao, O. Edfors, F. Rusek, and F. Tufvesson, "Massive MIMO performance evaluation based on measured propagation data," IEEE Trans. Wireless Commun., vol. 14, no. 7, Jul. 2015.

[15] J. Chani-Cahuana, C. Fager, and T. Eriksson, "A new variant of the indirect learning architecture for the linearization of power amplifiers," in European Microwave Conference (EUMC), Paris, 2015, pp. 12951298.

[16] W. H. Doherty, "A new high efficiency power amplifier for modulated waves," in Proceedings of the Institute of Radio Engineers, vol. 24, no. 9, pp. 1163-1182, Sept. 1936.

[17] H. Chireix, "High power outphasing modulation," in Proceedings of the Institute of Radio Engineers, vol. 23, no. 11, pp. 1370-1392, Nov. 1935.

[18] R. Hou, M. Spirito, J. Gajadharsing and L. C. N. de Vreede, "Nonintrusive characterization of active device interactions in high-efficiency power amplifiers," 2013 IEEE MTT-S International Microwave Symposium Digest (MTT), Seattle, WA, 2013, pp. 1-3.

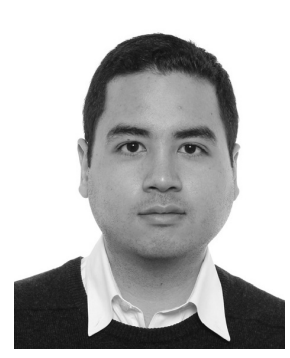

Dhecha Nopchinda (S'12) was born in Bangkok, Thailand, in 1991. He was awarded a scholarship to study at Sirindhorn International Institute of Technology, Thammasat University, Thailand, where he received his BEng (first-class honours) in electronics and communication engineering in 2013. He then continued his study in wireless and optical communications at University College London, United Kingdom, where he received his MSc (distinction) in 2014.

Since 2015, he has joined the Microwave Electronics Laboratory, Chalmers University of Technology, Sweden, as a $\mathrm{PhD}$ student under main supervision of Prof. Herbert Zirath. His research is on digital techniques for high data-rate communication at millimeter-wave.

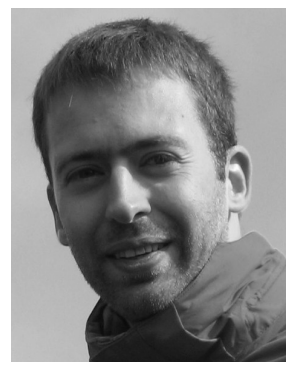

Koen Buisman (S'05-M'09) received the M.Sc. and Ph.D. degrees in microelectronics from the Delft University of Technology, Delft, The Netherlands, in 2004 and 2011, respectively.

From 2004 to 2014, he was with the Delft Institute of Microsystems and Nanoelectronics, Delft University of Technology. In 2014, he joined the Chalmers University of Technology, Gothenburg, Sweden, where he is currently an Assistant Professor with the Microwave Electronics Laboratory, Department of Microtechnology and Nanoscience.

He has authored or co authored over 50 refereed journal and conference papers. He holds two patents. His current research interests include nonlinear device characterization, technology optimization, and design of linear receivers for wireless systems. 